\title{
Maturation of the Central Auditory Nervous System in Children with Auditory Processing Disorder
}

\author{
Dani Tomlin, Ph.D. ${ }^{1}$ and Gary Rance, Ph.D. ${ }^{1}$
}

\section{ABSTRACT}

Neurodevelopmental delay has been proposed as the underlying cause of the majority of cases of auditory processing disorder (APD). The current study employs the cortical auditory evoked potential (CAEP) to assess if maturational differences of the central auditory nervous system (CANS) can be identified between children who do and do not meet the diagnostic criterion for APD. The P1-N1 complex of the CAEP has previously been used for tracking development of the CANS in children with hearing impairment. Twenty-seven children (7 to 12 years old) who failed an APD behavioral test battery were agematched (within 3 months) to children who had passed the same battery. CAEP responses to $500-\mathrm{Hz}$ tone burst stimuli were recorded and analyzed for latency and amplitude measures. The P1-N1 complex of the CAEP, which has previously been used for tracking development of the CANS in children with hearing impairment, showed significant group differences. The children diagnosed with APD showed significantly increased latency $(\sim 10$ milliseconds) and significantly reduced amplitude $(\sim 10 \mu \mathrm{V})$ of the early components of the CAEP compared with children with normal auditory processing. No significant differences were seen in the later $\mathrm{P} 2$ wave. The normal developmental course is for a decrease in latency and increase in amplitude as a function of age. The results of this study are, therefore, consistent with an immaturity of the CANS as an underlying cause of APD in children.

KEYWORDS: Cortical auditory evoked potential, central auditory nervous system development, auditory processing disorder

\footnotetext{
${ }^{1}$ Department of Audiology and Speech Pathology, the University of Melbourne, Parkville, Australia. Address for correspondence: Dani Tomlin, Ph.D., Department of Audiology and Speech Pathology, The University of Melbourne, 550 Swanston St., Parkville, Australia, 3010 (e-mail: dtomlin@unimelb.edu.au).
}

The Use of Cortical Auditory Evoked Potentials in Diagnosis and Treatment of Hearing Disorders; Guest Editor, Bram Van Dun, Ph.D.

Semin Hear 2016;37:74-83. Copyright (C) 2016 by Thieme Medical Publishers, Inc., 333 Seventh Avenue, New York, NY 10001, USA. Tel: +1(212) 584-4662. DOI: http://dx.doi.org/10.1055/s-0035-1570328. ISSN 0734-0451. 
Learning Outcomes: As a result of this activity, the participant will be able to identify the components of the cortical auditory evoked potential that relate to maturational changes of the central auditory nervous system and how children diagnosed with an auditory processing disorder show differences at the cortical level compared with children demonstrating normal auditory processing ability.

Children displaying behaviors synonymous with listening difficulties often will be referred for assessment of, and may subsequently be diagnosed with, an auditory processing disorder (APD). APD is an umbrella term that reflects poor performance within a behavioral test battery. ${ }^{1-3}$ Although no specific behavioral profiles align with a diagnosis of $\mathrm{APD},{ }^{4,5}$ behaviors such as difficulty hearing speech in background noise or concerns with attention, literacy, or spoken language often are reported. ${ }^{4,5}$ Significant associations of cognitive ability and a diagnosis of APD have been demonstrated. ${ }^{1,6-12}$

The etiology that causes children to perform poorly on auditory processing (AP) tasks, to such a degree that a diagnosis of APD occurs, is unknown; however, one theory put forward is of a maturational delay of the central nervous system (CNS). ${ }^{13}$ The 2011 British Society of Audiology position statement on APD describes the underlying cause responsible for the majority of cases of APD as a neurodevelopmental disorder, with associated cognitive, learning, and language deficits. ${ }^{14}$ Age effects are common to AP tests with studies generating normative data for AP tests showing improvement across age brackets, until adolescence is reached. ${ }^{8,15-20}$ Normal developmental changes over time allow for the presence of developmental delays and support the possibility of APD as a neuromaturational or developmental disorder.

The normal development of the central auditory system, or thalamic-cortical maturation, follows a similar course to the maturation of AP skills, ${ }^{21}$ with the superficial layers of the auditory cortex not maturing until adolescence. $^{21,22}$ Although evidence of myelination is present throughout the cortical layers by 6 years of age, increased myelination of the neural pathways continues into adolescence. ${ }^{23}$ This myelination allows for more rapid transmission within and between hemispheres and between cortical structures. Dendritic branch- ing structures also continue to mature in complexity until 12 years of age. ${ }^{23}$

The maturation of the auditory cortex is demonstrated by changes to the cortical auditory evoked potential (CAEP) from birth into adulthood, including changes to the latencies, amplitude, and morphology of the CAEP over time. ${ }^{2,24,25}$ The early components of the CAEP waveform, P1 and N1, have multiple cortical sources that encode the auditory stimulus. They are passive responses that can be reliably elicited in children, unlike the later components of the waveform, which are performance-related and influenced by factors such as attention. ${ }^{24}$ The $\mathrm{P} 2$ and later components of the CAEP are not thought to be generated by the auditory cortex but by several sensory modalities. ${ }^{22}$ Maturational changes to latency and amplitude are documented for the $\mathrm{P} 1$ and N1 components, showing decreased latency and increased amplitude into adolescence, ${ }^{24,25}$ whereas no significant age-related changes have been noted for the P2 marker. ${ }^{22}$

The P1 component of the CAEP has been extensively used as a biomarker of maturity of the CANS. ${ }^{24,26-31}$ The P1 component of the CAEP is a robust obligatory positivity occurring at 50 to 150 milliseconds after stimulus onset that is present in infancy, ${ }^{24,25}$ unlike the later components that emerge during childhood. The P1 is generated by the auditory thalamic and cortical regions of the CANS and shows decreased latency and increased amplitude into adolescence. ${ }^{24,25}$ This systematic decrease in latency has allowed the P1 potential to be used as a biomarker of central auditory development. In particular, the P1 biomarker has been employed for hearing-impaired children using maturation of the central auditory nervous system as an indicator of the effectiveness of interventions (such as cochlear implantation or hearing aid use). ${ }^{24,26-31}$

Few studies have examined CAEPs in children clinically diagnosed with APD. Sharma et al demonstrated a trend of increased 
latency and decreased P1 amplitude for a group of children diagnosed with APD $(n=55$, age 9 to 12 years) and control group ( $n=22$, age 7 to 12 years) to a speech phoneme presented in quiet at a rate of $\sim 1.1 / \mathrm{s} .{ }^{32}$ Only the amplitude result was shown to be significantly different. Purdy et al examined CAEP results in a group of children $(n=10)$ diagnosed with learning disabilities and suspected of having an APD. ${ }^{33}$ An amplitude effect was demonstrated at N1, with larger amplitudes shown in a control group ( $n=10$, age 7 to 11 years), which is consistent with immaturity of the CAEP in the suspected APD group; however, the latency effect found was not. The children with learning disabilities showed shorter latencies of the P1 component compared with the control group, which is inconsistent with immaturity but does show that cortical differences are present in this group. An oddball paradigm was employed in this study: the child attended to the deviant (20\% of presentations) $2-\mathrm{kHz}$ tone bursts presented at a rate of $1.1 / \mathrm{s}$, with the standard stimulus of a $1-\mathrm{kHz}$ tone burst, to elicit the CAEP. Previous studies that have explored maturational changes to the CAEP have not used an oddball paradigm, but elicited an obligatory response, with no task associated. ${ }^{2,24,25}$ Although the children in the Purdy et al study were suspected of having an APD, no clinical diagnosis was made. ${ }^{33}$ Age-matched controls were reported, but it is not stated within how many months of age they were matched.

Normally occurring maturational changes are a complication when comparing CAEP differences in children diagnosed with APD with control groups. Group differences may be masked unless age is equally balanced across the group under investigation. Sample sizes also need to be large enough to allow the effect to be seen. No previous studies have aimed to examine maturational CAEP differences in children clinically diagnosed with an APD, using agematched comparisons. Where differences exist in CAEP findings between test and control groups, samples sizes are small or children are not age-matched. Subject characteristics also need to be clearly defined, such as how the diagnosis of APD was made; many studies have shown that children suspected of having an
APD have normal AP skills when clinically assessed. ${ }^{4,34}$ If evidence of a maturational delay in children with APD becomes evident from CAEP results, the test paradigm employed for the CAEP must have been previously demonstrated to show maturational changes in typically developing children. Furthermore, these normal maturational changes must be controlled for, and the cohort of children for whom a diagnosis of APD is in place must be well defined.

\section{AIM}

This study examines the maturation of the CANS, as measured by the CAEP, in children who have been clinically identified as having an APD. Evidence of CAEP differences (specifically at the markers that have been demonstrated to undergo maturational changes), in comparison to age-matched pairs, would support a definition of APD as a neurodevelopmental disorder.

\section{METHODS}

This study was conducted with the ethical approval and oversight of the Royal Victorian Eye and Ear Hospital Human Research Ethics Committee.

\section{Participants}

Children were recruited from those referred to the University of Melbourne Audiology clinic for an AP assessment. Children were assessed using a clinical test battery including tests of AP and CAEPs. All children had normal peripheral hearing thresholds $(15-\mathrm{dB}$ hearing loss [HL] or better, bilaterally, at each octave frequency in the $250-\mathrm{Hz}$ to $8-\mathrm{kHz}$ range) and normal type A tympanograms. Assessment occurred over two sessions. Repeatable auditory brainstem responses also were recorded in all children. All demonstrated interpeak latencies within normal range (waves I to $\mathrm{V}$ conduction time $<4.2$ milliseconds for an $11.1-\mathrm{Hz}$ click presentation rate). This result was interpreted as being consistent with normal conduction of the VIII nerve and auditory brainstem responses. All children also showed nonverbal 
intelligence quotient scores above 85 (see below).

\section{Auditory Processing Assessments}

Five AP tasks were used including the Dichotic Digits Test-2, The Frequency Pattern Test, ${ }^{19,35}$ Gaps in Noise, ${ }^{36}$ Masking Level Differences, ${ }^{37}$ and the Listening in Spatialized Noise-Sentences Test. ${ }^{38}$ This battery represents tasks that are commonly used in the clinical setting, ${ }^{39}$ and it is in alignment with the recommendations of the American Academy of Audiology. ${ }^{40}$ Test protocols were as described in Tomlin et al. ${ }^{34}$ Twenty-seven children (16 boys, 11 girls) age 7.2 to 11.9 years of age (mean 9.03 years, standard deviation [SD] 1.43) were diagnosed with APD when performance was $\geq 2 \mathrm{SD}$ below the mean on one or more of the behavioral APD tests. This group of children comprised the APD+ group. Twenty-seven additional children (16 boys, 11 girls; 7.2 to 11.6 years of age, mean 8.99 years, SD 1.34) who had passed the same AP test battery were then identified from the same cohort as age-matched pairs (within 3 months of age). The group was the APD - .

Measures of nonverbal intelligence, sustained attention, and auditory working memory also were collected to explore group differences. Nonverbal intelligence was assessed using the normreferenced Test of Non Verbal Intelligence $4 .^{41}$ Auditory memory was assessed using the Clinical Evaluation of Language Fundamentals, Fourth Edition forward and reverse digit tasks. ${ }^{42}$ The Integrated Visual and Auditory Continuous Performance Test was used to assess sustained attention. These assessments are described in detail elsewhere ${ }^{34}$ Cognitive findings for the AP deficit $(\mathrm{APD}+)$ and control (APD - ) groups are described in Table 1.

\section{Cortical Auditory Evoked Potential Assessment}

The CAEP testing was performed in a soundproof booth using the Biologic AEP (Natus Medical Incorporated, San Carlos, CA) system to deliver stimuli and record responses. Children were given a handheld tablet to play on quietly for the duration of the assessment and were asked to sit quietly. Obligatory CAEPs were recorded to $500-\mathrm{Hz}$ tone bursts presented at $80-\mathrm{dB}$ normalized hearing Level (nHL) monaurally to left and right ears via ER3-A insert (Etymotic Research, Elk Grove Village, IL). The tone burst duration was 100 milliseconds (80-millisecond plateau with 10-millisecond rise and fall times), presented at $0.3 / \mathrm{s}$. It was predicted that at this slow rate the earlier components of the waveform would be evident across the age group. ${ }^{43}$ CAEPs were recorded form vertex $(\mathrm{Cz})$ midline electrodes, reference electrodes on left and right mastoids and ground electrode on high forehead. Responses were recorded for 40 averages and two waveforms collected. The electroencephalogram was filtered $100 \mathrm{~Hz}$ to $3,000 \mathrm{~Hz}$. The components of the waveform were identified visually by an experienced clinician (blinded to subject categorization). The $\mathrm{P} 1$ and $\mathrm{N} 1$ components of the CAEP waveform were present in all children assessed. Peak-to-peak amplitude measures were obtained for P1-N1 and N1-P2. An example of an age matched pair of waveforms is shown in Figure 1.

\section{RESULTS}

Latency and amplitude results are shown in Table 2. No significant difference $(p>0.6)$ between ears was found for all measures using paired $t$ test comparisons. Results are therefore expressed as the average of both ears. Significant group differences in latency were found using the paired $t$ test (Fig. 2). The P1 latency was found to be significantly shorter (mean difference of 8.39 milliseconds, SD $13.12)$ in the APD- group $(p=0.03$, $T=3.32,95 \%$ confidence interval $=3.20$, 13.58). The N1 latency was also significantly shorter (mean difference of 9.63 milliseconds, $\mathrm{SD} 17.26)$ in the APD- group $(p>0.01$, $T=2.90,95 \%$ confidence interval $=2.80$, 16.46). No significant difference $(p=0.89)$ was found for P2 latency between the APDand APD+ groups.

Significant group differences in amplitude were also evident. A paired $t$ test showed significantly reduced amplitude (mean difference of $-4.16 \mathrm{~V}, \mathrm{SD} 6.70$ ) for the APD+ group in comparison with the APD- group 
Table 1 Cognitive Results of the APD + and APD- Groups

\begin{tabular}{|c|c|c|c|c|}
\hline & APD + & APD- & $\begin{array}{l}\text { Paired } \boldsymbol{t} \text { test results (95\% } \\
\text { confidence interval) }\end{array}$ & $p$ Value \\
\hline $\begin{array}{l}\text { Attention } \\
\text { (quotient score) }\end{array}$ & $72.68(22.35)$ & $90.16(22.20)$ & $\mathrm{T}=-2.78(-30.68,-4.27)$ & 0.012 \\
\hline $\begin{array}{l}\text { Working memory } \\
\text { (scaled score) }\end{array}$ & $7.98(1.76)$ & $9.83(2.25)$ & $\mathrm{T}=-2.97(-3.13,-0.56)$ & 0.007 \\
\hline $\begin{array}{l}\text { Nonverbal IO } \\
\text { (quotient score) }\end{array}$ & $99.44(8.73)$ & 104.00 (8.89) & $\mathrm{T}=-1.97(-9.34,0.22)$ & 0.06 \\
\hline
\end{tabular}

Abbreviations: APD + , group with auditory processing disorder; APD - , group without auditory processing disorder.

Table 2 Latency and Amplitude Results for P1, N1, and P2 Measures

\begin{tabular}{|c|c|c|c|c|c|c|c|}
\hline & \multicolumn{3}{|l|}{ Latency (ms) } & \multicolumn{4}{|c|}{ Amplitude (uV) } \\
\hline & $\mathrm{APD}+$ & APD- & & & APD + & APD- & \\
\hline P1 & $90.41(9.43)$ & 82.02 (8.32) & * & P1-N1 & $9.36(3.86)$ & $12.75(4.94)$ & * \\
\hline N1 & $129.04(11.43)$ & $119.41(14.28)$ & * & & & & \\
\hline P2 & $174.62(18.85)$ & $173.86(23.12)$ & NS & N1-P2 & 12.107 .07 & $12.29(7.86)$ & NS \\
\hline
\end{tabular}

Abbreviations: APD + , group with auditory processing disorder; APD - , group without auditory processing disorder; NS, not significant.

Note: Results are shown as mean (standard deviation). ${ }^{*} p<0.05$.

$(p=0.03, T=-2.4,95 \%$ confidence interval $=-7.87,-0.45)$. No significant difference $(p=0.89)$ was found for N1-P2 amplitude between the two groups.
Significant differences also were found in the cognitive scores of the APD+ and APDgroups, as shown in Table 1. These group differences are consistent with reported associ-

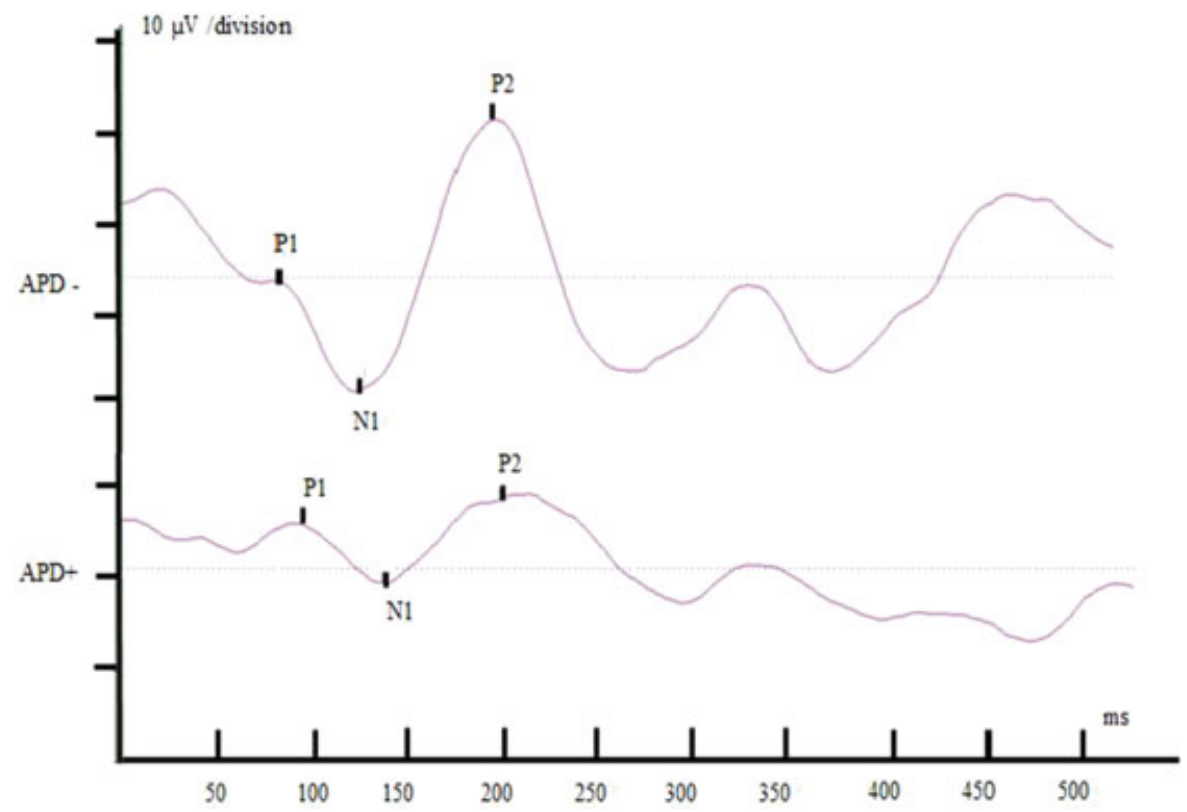

Figure 1 Cortical auditory evoked potential of two age-matched children: one without (top) and one with auditory processing disorder (APD) (bottom). 


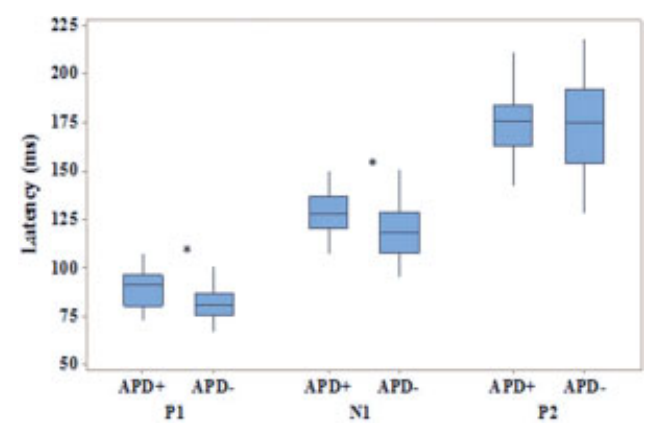

Figure 2 Latency results of the groups with auditory processing disorder (APD + ) and without APD $(\mathrm{APD}-)$, showing interquartile range. ${ }^{*} p<0.05$.

ation of cognition and APD. For further discussion of this association, see Tomlin et al. ${ }^{34}$

\section{DISCUSSION}

The aim of this study was to determine if CAEP differences were evident in children diagnosed with an APD and if these differences were consistent with CANS immaturity. The early P1-N1 components of the CAEP waveform were found to be significantly later in latency (a mean difference of $\sim 10$ milliseconds) and smaller in amplitude (a mean difference of $\sim 5 \mathrm{~V}$ ) in the group of children who met the diagnostic criterion for the presence of an APD in comparison to age-matched peers. These waveform morphology differences are consistent with the well-documented maturational changes to the waveform that occur into adolescence: latency decrease and amplitude increase of the earlier P1-N1 complex and no significant latency or amplitude changes for the later P2 component. ${ }^{22,24,25}$ These results are consistent with a delayed maturation of the CANS in children diagnosed with an APD. Neural dysfunction causing conduction times to be slowed in the auditory pathway cannot be ruled out, but the later waves (although generated by multiple sensory sites) would also be expected to show a delayed effect. A longitudinal study would demonstrate the rate of maturation occurring and provide stronger evidence of a delayed maturation pattern.

Differences at the P1 marker in APD versus control groups has been demonstrated previously; however, this was a reduced amplitude of response only. ${ }^{32,33}$ A significant latency increase in children diagnosed with APD, as is seen in the current study, has not previously been reported. Aside from stimulus differences, a possible explanation as to why different results are present between the current study and Sharma et al is the comparisons made. ${ }^{32}$ Although Sharma et al compared group difference between the APD children and a control group, age was not controlled for. ${ }^{32}$ Children were not in age-matched pairs and the age ranges differed between the two groups; the control group ranged from 7 to 12 years, and the test group ranged from 9 to 12 years. It is possible that the latency effects seen in the current study are now evident because normal maturational changes are being tightly controlled for. Purdy et al also demonstrated an amplitude effect consistent with immaturity but not latency (in fact the opposite latency effect was found). ${ }^{33}$ Purdy et al had a small sample size and employed an oddball paradigm, which may explain the different findings. ${ }^{33}$ Furthermore, the children were suspected of APD but not clinically diagnosed as being an APD group. The children in the present study are closely age-matched and differentiated by a clinical diagnosis of APD; their ability to complete the AP test battery defined the group in which they fell.

Strong associations are reported between a child's cognitive abilities (auditory working memory and attention) and performance on AP tasks, ${ }^{1,6-12}$ and this is again evident in the current cohort, with the APD+ group showing significantly poorer auditory working memory and attention skills than the APD - group. This association of cognition and APD is consistent with the CAEP differences. The maturation of the CNS parallels normal development of cognitive skills. Cerebral maturation and cognitive development are interdependent processes, and stages of cognitive development have associated stages of myelination and dendritic branching throughout childhood and into adolescence. ${ }^{23}$ Electroencephalogram studies of CNS maturation demonstrate several growth periods, including one from 7 to 9 years of age associated with strengthening of cortical synaptic connections. ${ }^{44,45}$ Models are of stepwise development 
of the CNS, rather than a gradual progression, with evidence that growth spurts occur in early infancy, again around 7 to 10 years of age, ${ }^{46}$ and with a final spurt during adolescence. The rate of development of cognitive skills has also been demonstrated in both working memory and sustained attention tasks with significant periods of development occurring between 7 and 10 years of age. ${ }^{47-49}$ So too many AP tasks show significant developmental changes in the 7- to 10-year bracket mirroring that seen in the CNS and cognitive models of development. ${ }^{8,15-20}$ Rather than the assumed causal link of cognitive ability driving a child's performance on behavioral AP tasks, an alternative explanation is that performance on behavioral tasks (whether designed to assess cognitive skills or AP ability) are a reflection the overall development of the CANS, in line with the theory of APD as a neurodevelopmental disorder. The line of causality may of course run in the other direction with a peripheral dysfunction causing a delay in cortical and cognitive maturation also. Regardless, differences (that are consistent with delays of maturation) are evident at the cortical level in children who demonstrate difficulty performing AP tasks.

It is relevant to note that APD reflects a variety of potential dysfunctions in the auditory system. The term auditory processing disorder does not specify on which tasks in the battery the child performed sufficiently poorly for a diagnosis to occur. In this current cohort, the majority of children were found to have a deficit in either the Frequency Pattern Test (FPT) or Dichotic Digits test (DDT) tasks, or both. These two behavioral tasks in particular require interhemispheric transfer of information. ${ }^{15,50}$ The maturation of the CANS includes a process of increasing myelination, which allows for a more rapid transmission of information between the hemispheres. ${ }^{23}$ It is evident in this current study that as a group, the children who fall above the diagnostic criterion demonstrate CAEP waveforms consistent with more advanced development than those who fall below.

The clinical application of this CAEP difference is yet to be developed. What is not evident in the present study is whether the observed latency differences are significant enough that children with an APD would fall outside of the normal range of latencies, allowing for the CAEP to be employed as a diagnostic tool. Studies that have tracked the development of P1 latency following cochlear implantation report a "normal" range of latencies of $\sim 50$ milliseconds using speech stimuli in the 6- to 10 -year age range. ${ }^{30}$ The mean latency differences in this current study are of $\sim 10$ milliseconds, which may be not be sufficiently different to allow for clinical differentiation. Large-scale normative data are required to answer this question. This study has employed a simple tone stimuli in quiet, with long interstimulus interval; different stimulus characteristics including the use of speech stimuli and speech in noise stimuli may be more (or less) sensitive to these maturational changes.

Evidence, such as is seen in the current study, that supports the theory of neurodevelopmental delay in children with APD also supports the application of auditory training programs as a management tool for the disorder. Auditory training programs target the neural plasticity of the CANS. The CANS is not mature until adolescence, and enriched stimulation has been reported to increase maturational rates. ${ }^{51}$ Evidence of immaturities provides a framework for training programs to accelerate the normal maturational process. Whether the P1 marker has the necessary sensitivity to objectively monitor the success of auditory training programs has yet to be seen. This tracking of the P1 latency has been employed in hearing impaired children to measure rehabilitation progress ${ }^{26-28}$; however, this is following significant auditory deprivation and for young children in whom more pronounced maturational changes to the CAEP are documented compared with the 7- to 12-year age group where a more subtle rate of development is reported. ${ }^{30}$ CAEP changes have been reported post-auditory training in children with APD, although these have been in the later wave components, which are influenced by cognitive ability and task-specific behaviors, such as attention to stimulus, but not in P1 latency. ${ }^{32}$ Furthermore, whether CAEP changes indicate significant real-life differences in ability also needs to be determined. 


\section{CONCLUSIONS}

The results of the present study show that cortical differences are present in children who perform poorly on AP tasks. Significant differences are seen of increased P1 and N1 latencies and reduced P1-N1amplitude in children diagnosed with an APD. Latency decrease and amplitude increase of the $\mathrm{P} 1$ and $\mathrm{N} 1$ waves of the CAEPs are maturational markers of the CAEP and the CANS. This pattern of difference is consistent with immaturity of the CANS in the children who meet the diagnostic criterion for APD and a description of APD as a neurodevelopmental disorder. The neuromaturational delay in children with APD is consistent with the documented association of cognitive and AP abilities. This has implications for the future development of test batteries and remediation programs. Many causes of listening difficulties are likely to exist, but for this cohort of children, who meet the clinical diagnostic criterion for the presence of APD, cortical differences consistent with immaturity of the CANS are evident. For these children, an objective measure defining the cause of the listening deficit would allow for the application of targeted, appropriate rehabilitation.

\section{REFERENCES}

1. Riccio CA, Hynd GW, Cohen MJ, Hall J, Molt L. Comorbidity of central auditory processing disorder and attention-deficit hyperactivity disorder. J Am Acad Child Adolesc Psychiatry 1994;33(6): 849-857

2. Bellis T. Assessment and Management of Central Auditory Processing Disorders in the Educational Setting from Science to Practice, 2nd ed. New York, NY: Delmar Learning, Thompson Learning; 2003

3. Geffner D, Ross-Swain D. Auditory Processing Disorders: A Handbook for Management and Treatment for Speech-language Pathologists. San Diego: Plural Publishing; 2007

4. Rosen S, Cohen M, Vanniasegaram I. Auditory and cognitive abilities of children suspected of auditory processing disorder (APD). Int J Pediatr Otorhinolaryngol 2010;74(6):594-600

5. Dawes P, Bishop DV, Sirimanna T, Bamiou DE. Profile and aetiology of children diagnosed with auditory processing disorder (APD). Int J Pediatr Otorhinolaryngol 2008;72(4):483-489

6. Cook JR, Mausbach T, Burd L, et al. A preliminary study of the relationship between central auditory processing disorder and attention deficit disorder. J Psychiatry Neurosci 1993;18(3):130-137

7. Ferguson MA, Hall RL, Riley A, Moore DR. Communication, listening, cognitive and speech perception skills in children with auditory processing disorder (APD) or Specific Language Impairment (SLI). J Speech Lang Hear Res 2011; 54(1):211-227

8. Moore DR, Cowan JA, Riley A, EdmondsonJones AM, Ferguson MA. Development of auditory processing in 6- to 11-yr-old children. Ear Hear 2011;32(3):269-285

9. Sutcliffe PA, Bishop DV, Houghton S, Taylor M. Effect of attentional state on frequency discrimination: a comparison of children with ADHD on and off medication. J Speech Lang Hear Res 2006; 49(5):1072-1084

10. Ferguson MA, Moore DR. Auditory processing performance and nonsensory factors in children with specific language impairment or auditory processing disorder. Semin Hear 2014;35(1):1-14

11. Gyldenkærne P, Dillon H, Sharma M, Purdy SC. Attend to this: the relationship between auditory processing disorders and attention deficits. J Am Acad Audiol 2014;25(7):676-687, quiz 706-707

12. Wilson WJ, Jackson A, Pender A, et al. The CHAPS, SIFTER, and TAPS-R as predictors of (C)AP skills and (C)APD. J Speech Lang Hear Res 2011;54(1):278-291

13. Musiek FE, Gollegly KM, Ross MK. Profiles of types of central auditory processing disorders in children with learning disabilities. Comm Disord Q1985;9(1):43-63

14. British Society of Audiology. Position Statement on Auditory Processing Disorder (APD). 2011; http://www.thebsa.org.uk/resources/apd-positionstatement/. Accessed December 16, 2015

15. Baran J, Musiek F. Behavioural assessment of the central auditory nervous system. In: Musiek F, Rintelmann W, eds. Contemporary Perspectives in Hearing Assessment. Boston, MA: Allyn and Bacon; 1999:375-414

16. Bellis T. Assessment and Management of Central Auditory Processing Disorders in the Educational Setting. From Science to Practice. San Diego, CA: Singular Publishing Group; 1996

17. Kelly AS. Normative data for behavioural tests of auditory processing for New Zealand school children aged 7-12 years. Aust N Z J Audiol 2007; 29(1):60-64

18. Singer J, Hurley RM, Preece JP. Effectiveness of central auditory processing tests with children. Am J Audiol 1998;7(2):73-84 
19. Musiek FE. Frequency (pitch) and duration pattern tests. J Am Acad Audiol 1994;5(4):265-268

20. Tomlin D, Dillon H, Kelly AS. Allowing for asymmetric distributions when comparing auditory processing test percentage scores with normative data. J Am Acad Audiol 2014;25(6):541-548

21. Eggermont JJ, Ponton CW. Auditory-evoked potential studies of cortical maturation in normal hearing and implanted children: correlations with changes in structure and speech perception. Acta Otolaryngol 2003;123(2):249-252

22. Ponton CW, Eggermont JJ, Kwong B, Don M. Maturation of human central auditory system activity: evidence from multi-channel evoked potentials. Clin Neurophysiol 2000;111(2):220-236

23. Hallett T, Proctor A. Maturation of the central nervous system as related to communication and cognitive development. Infants Young Child 1996; 8(4):1-15

24. Sharma A, Kraus N, McGee TJ, Nicol TG. Developmental changes in P1 and N1 central auditory responses elicited by consonant-vowel syllables. Electroencephalogr Clin Neurophysiol 1997; 104(6):540-545

25. Wunderlich JL, Cone-Wesson BK, Shepherd R. Maturation of the cortical auditory evoked potential in infants and young children. Hear Res 2006; 212(1-2):185-202

26. Dorman MF, Sharma A, Gilley P, Martin K, Roland P. Central auditory development: evidence from CAEP measurements in children fit with cochlear implants. J Commun Disord 2007;40(4): 284-294

27. Nash A, Sharma A, Martin K, Biever A. Clinical applications of the $\mathrm{P} 1$ cortical auditory evoked potential (CAEP) biomarker. Paper presented at: A Sound Foundation through Early Amplification: Proceedings of a Fourth International Conference; 6th December, 2007; Chicago, IL

28. Sharma A, Dorman MF, Spahr AJ. Rapid development of cortical auditory evoked potentials after early cochlear implantation. Neuroreport 2002; 13(10):1365-1368

29. Sharma A, Gilley PM, Dorman MF, Baldwin R. Deprivation-induced cortical reorganization in children with cochlear implants. Int $\mathrm{J}$ Audiol 2007;46(9):494-499

30. Sharma A, Glick H, Campbell J, Biever A. Central auditory development in children with hearing impairment: clinical relevance of the P1 CAEP biomarker in children with multiple disabilities. Hearing Balance Commun. 2013;11(3): 110-120

31. Sharma A, Martin K, Roland P, et al. P1 latency as a biomarker for central auditory development in children with hearing impairment. J Am Acad Audiol 2005;16(8):564-573
32. Sharma M, Purdy SC, Kelly AS. The contribution of speech-evoked cortical auditory evoked potentials to the diagnosis and measurement of intervention outcomes in children with auditory processing disorder. Semin Hear 2014;35(1):51-64

33. Purdy SC, Kelly AS, Davies MG. Auditory brainstem response, middle latency response, and late cortical evoked potentials in children with learning disabilities. J Am Acad Audiol 2002;13(7):367-382

34. Tomlin D, Dillon H, Sharma M, Rance G. The impact of auditory processing and cognitive abilities in children. Ear Hear 2015;36(5):527-542

35. Noffsinger D, Wilson RH, Musiek FE. Department of Veterans Affairs compact disc recording for auditory perceptual assessment: background and introduction. J Am Acad Audiol 1994;5(4):231-235

36. Musiek FE, Shinn JB, Jirsa R, Bamiou DE, Baran JA, Zaida E. GIN (Gaps-In-Noise) test performance in subjects with confirmed central auditory nervous system involvement. Ear Hear 2005;26(6):608-618

37. Aithal V, Yonovitz A, Aithal S. Tonal masking level differences in aboriginal children: implications for binaural interaction, auditory processing disorders and education. Aust N Z J Audiol 2006;28(1):31-40

38. Cameron S, Dillon H. Development of the Listening in Spatialized Noise-Sentences Test (LISNS). Ear Hear 2007;28(2):196-211

39. Sharma M, Purdy SC, Kelly AS. Comorbidity of auditory processing, language, and reading disorders. J Speech Lang Hear Res 2009;52(3):706-722

40. American Academy of Audiology, Clinical Practice Guidelines: Diagnosis treatment and management of children and adults with central auditory processing disorder. 2010; Available at: http://audiology-web.s3.amazonaws.com/migrated/CAPD Guidelines 8-2010.pdf_539952 af956c79.73897613.pdf. Last accessed December 2015

41. Brown L, Sherbenou R, Johnsen KJ. Test of NonVerbal Intelligence. 4th ed. Austin, TX: Pro-Ed; 2010

42. Semel E, Wiig E, Secord W. Clinical Evaluation of Language Fundamentals. Fourth Edition (CELF-4). San Antonio, TX: The Psychological Corporation; 2003

43. Gilley PM, Sharma A, Dorman M, Martin K. Abnormalities in central auditory maturation in children with language-based learning problems. Clin Neurophysiol 2006;117(9):1949-1956

44. Thatcher RW. Maturation of the human frontal lobes: physiological evidence for staging. Dev Neuropsychol 1991;7(3):397-419

45. Thatcher RW. Cyclic cortical reorganization during early childhood. Brain Cogn 1992;20(1):24-50

46. Hudspeth WJ, Pribram KH. Stages of brain and cognitive maturation. J Educ Psychol 1990;82(4): 881-888 
47. Brocki KC, Bohlin G. Executive functions in children aged 6 to 13: a dimensional and developmental study. Dev Neuropsychol 2004;26(2):571-593

48. Towse JN, Hitch GJ, Hutton U. A reevaluation of working memory capacity in children. J Mem Lang 1998;39(2):195-217

49. Gale A, Lynn R. A developmental study of attention. Br J Educ Psychol 1972;42(3):260-266
50. Musiek FE, Baran JA. Central auditory assessment: thirty years of challenge and change. Ear Hear 1987;8(4, Suppl)22S-35S

51. Musiek F, Chermak G, Weihing J. Auditory training. In: Chermak GD, Musiek FE, eds. Handbook of (Central) Auditory Processing Disorder: Comprehensive Intervention. San Diego, CA: Plural Publishing; 2007:77-106 